\title{
An exploration of the control of micturition using a novel in situ arterially perfused rat preparation
}

\author{
Prajni Sadananda ${ }^{1}$, Marcus J. Drake ${ }^{2}$, Julian F. R. Paton ${ }^{1}$ and Anthony E. Pickering ${ }^{\text {* }}$ \\ ISchool of Physiology and Pharmacology, University of Bristol, Bristol, UK \\ 2 Bristol Urological Institute, Southmead Hospital, Bristol, UK
}

Edited by:

Margaret A. Vizzard, University of

Vermont College of Medicine, USA

Reviewed by:

Margaret A. Vizzard, University of

Vermont College of Medicine, USA

Chang Feng Tai, University of

Pittsburgh, USA

${ }^{*}$ Correspondence:

Anthony E. Pickering, Department of

Physiology and Pharmacology,

University of Bristol, Bristol BS8 1TD,

UK.

e-mail: tony.pickering@bristol.ac.uk
Our goal was to develop and refine a decerebrate arterially perfused rat (DAPR) preparation that allows the complete bladder filling and voiding cycle to be investigated without some of the restrictions inherent with in vivo experimentation [e.g., ease and speed of set up (30 min), control over the extracellular milieu and free of anaesthetic agents]. Both spontaneous (naturalistic bladder filling from ureters) and evoked (in response to intravesical infusion) voids were routinely and reproducibly observed which had similar pressure characteristics. The DAPR allows the simultaneous measurement of bladder intra-luminal pressure, external urinary sphincterelectromyogram (EUS-EMG), pelvic afferent nerve activity, pudendal motor activity, and permits excellent visualization of the entire lower urinary tract, during typical rat filling and voiding responses. The voiding responses were modulated or eliminated by interventions at a number of levels including at the afferent terminal fields (intravesical capsaicin sensitization-desensitization), autonomic (ganglion blockade with hexamethonium), and somatic motor (vecuronium block of the EUS) outflow and required intact brainstem/hindbrain-spinal coordination (as demonstrated by sequential hindbrain transections). Both innocuous (e.g., perineal stimulation) and noxious (tail/ paw pinch) somatic stimuli elicited an increase in EUS-EMG indicating intact sensory feedback loops. Spontaneous non-micturition contractions were observed between fluid infusions at a frequency and amplitude of $1.4 \pm 0.9$ per minute and $1.4 \pm 0.3 \mathrm{mmHg}$, respectively, and their amplitude increased when autonomic control was compromised. In conclusion, the DAPR is a tractable and useful model for the study of neural bladder control showing intact afferent signaling, spinal and hindbrain co-ordination and efferent control over the lower urinary tract end organs and can be extended to study bladder pathologies and trial novel treatments.

Keywords: bladder, external urinary sphincter, bladder afferent activity, brainstem, decerebrate, capsaicin, hexamethonium, voiding, incontinence

\section{INTRODUCTION}

The urinary cycle consists of two-phases of bladder activity: filling and voiding, which are under both voluntary and autonomic neural control. During filling, the external urinary sphincter (EUS) muscle is contracted, thus maintaining continence while the bladder is relatively relaxed and distends gradually to accommodate urine. During voiding, the activity of the EUS changes to allow the passage of urine and the detrusor muscle of the bladder exerts a coordinated contraction to expel urine. The pontine micturition center (PMC) and the sacral spinal cord are believed to be important in the spino-bulbarspinal coordinated phases of bladder-EUS control in response to inputs from bladder afferents (Sasaki, 2005; de Groat, 2006; Drake et al., 2010). However, we have an incomplete understanding of the neural mechanisms that generate and regulate the phases of micturition.

To date, the investigation of central neural control of the bladder has been hampered by the lack of suitable whole animal models that permit the simultaneous investigation of central and peripheral control of the bladder and EUS in the absence of anaesthesia. The majority of the commonly utilized animal models for autonomic bladder studies involve urethane anaesthetized animals, as other known anaesthetics suppress the micturition reflex (Maggi et al., 1986). Even with urethane, the depth of anaesthesia has a marked effect on whether the animal displays a voiding response (Conte et al., 1988; Maggi and Conte, 1990). Thus, acute studies of bladder function are technically challenging and often focus on the filling/storage mechanisms, since the voiding response is functionally inconsistent. In this respect, the alternative strategy of using conscious, telemetered animals has some advantages but the ability to study central neuronal control mechanisms is limited and recordings of peripheral afferents, although possible are non-trivial (Zvara et al., 2010).

The more common approach to these challenges has been to work with reduced, in vitro preparations to study molecular and cellular level mechanics of lower urinary tract function. Such in vitro studies have the advantage of good control over the extracellular environment and the ability to compare and contrast the effects of pharmacological agents and stimuli on the same tissue. This has led to significant advances in our understanding of the peripheral aspects of bladder function and its afferent innervation (Ferguson et al., 1997; Hawthorn et al., 2000; Avelino et al., 2002; Sadananda et al., 2008; Gillespie et al., 2009; Kanai, 2011). However it is challenging to appraise the importance of 
these molecular and cellular level insights in an integrated setting particularly given that the study of micturition requires intact central neural control.

Our goal was to develop an acute model that allows the complete filling and voiding cycle to be investigated in a preparation with:

- intact functional sensory and motor neural connectivity to end organs

- good access for recording, stimulation, and drug application

- some of the advantages of the in vitro preparations, e.g., ease of set up and control over the extracellular milieu

- no requirement for anaesthetic agents.

Our laboratory has described several artificially perfused in situ preparations for integrative physiological experiments including the working heart-brainstem preparation (Paton, 1996), the arterially perfused trunk-hindquarters preparation (Chizh et al., 1997) and the decerebrate artificially perfused whole rat preparation (Pickering and Paton, 2006). These decerebrate rodent preparations retain afferent and efferent connectivity and show robust central autonomic and motor functionality. Of these preparations the DAPR retains the pelvic viscera, ureters, and kidneys with intact bidirectional afferent-motor connections to the hindbrain (Pickering and Paton, 2006).

The aim of the present study was to develop and refine the DAPR for the study of bladder autonomic control. We demonstrate that the preparation has intact bladder afferent-brainstem-bladder motor circuitry, which allows strong and consistent filling and voiding responses lasting for up to $4 \mathrm{~h}$. This preparation permits the simultaneous measurement of bladder intra-luminal pressure, EUS-electromyogram (EMG), pelvic afferent nerve activity, pudendal motor activity, as well as recordings from respiratory nerves, ECG, and arterial pressure.

\section{MATERIALS AND METHODS}

All experiments conformed to the UK Home office guidelines regarding the ethical use of animals and were approved by our institutional ethical review committee.

\section{PREPARATION SET UP}

The procedures for the DAPR preparation were as previously described (Pickering and Paton, 2006) and are outlined here in brief (Figure 1). Female Wistar rats (40-90 g, P20-P28) were heparinized (100 IU i.p.) $20 \mathrm{~min}$ prior to being deeply anaesthetized with halothane until loss of paw withdrawal reflex. Following a midline laparotomy, the stomach, spleen, and free intestine were vascularly isolated with ligatures and removed. This allowed good access to the bladder, kidneys, and ureters. The animal was then immediately cooled by immersion in carbogenated Ringer's $\left(4^{\circ} \mathrm{C}\right.$, composition below) and decerebrated, by aspiration, at the precollicular level to render it insentient (at this point the halothane was withdrawn).

The preparation was then skinned and pinned on a sylgard covered dissecting dish on ice. A midline sternotomy, with insertion of a spreading retractor, allowed access to the heart and lungs. The left phrenic nerve was identified and the lungs were carefully removed, taking care to leave the phrenic intact. Both atria were

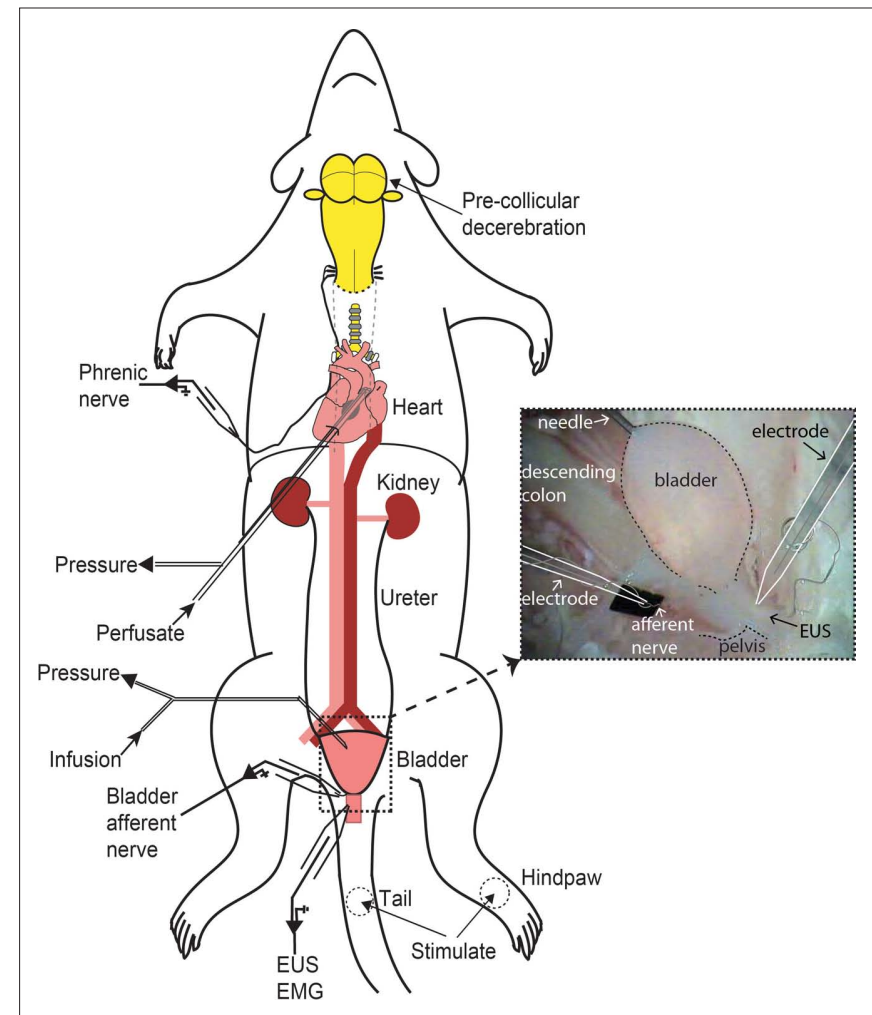

FIGURE 1 | Schematic of decerebrate arterially perfused rat (DAPR) in situ preparation for bladder studies. Shows the preparation with a double lumen cannula inserted via the left ventricle into the ascending aorta, allowing perfusate to be pumped into the arterial tree, as well as allowing continuous monitoring of perfusion pressure. Recording of phrenic nerve activity was used as a physiological indicator of brainstem viability. A needle (30G) inserted into the bladder dome allowed infusion of fluid and monitoring of intravesical pressure (see inset photograph of filled bladder). Simultaneous recordings of EUS-EMG activity and bladder afferent nerve were possible. Naturalistic stimuli could also be applied to the perineum, tail, or hindlimbs to evoke somatic and autonomic responses.

incised to avoid venous pressure build up during subsequent arterial perfusion. An incision was made at the apex of the heart for insertion of the perfusion cannula into the ascending aorta. The left phrenic nerve was detached from the diaphragm, which was then resected.

The preparation was transferred into the recording chamber and positioned supine with or without insertion of ear bars. The ear bars allowed for head rotation/flexion when access to the brainstem was required. A double lumen cannula ( $\varnothing 1.25 \mathrm{~mm}, \mathrm{DLR}-4$, Braintree Scientific, MA, USA) was inserted under direct vision using a dissecting microscope, into the ascending aorta. The cannula was held in place by a ligature. The time taken from the start of surgery to establishing perfusion was typically $20 \mathrm{~min}$, however more complex surgical preparation could be carried out for up to an hour, provided the preparation was kept cold during this time before perfusion was commenced.

The preparation was arterially perfused with carbogen-gassed, Ringer's, containing Ficoll-70 (1.25\% Sigma), at $32^{\circ} \mathrm{C}$. The perfusate was pumped from a reservoir flask, via a heated water bath, through two bubble traps and a particle filter $(25 \mu \mathrm{m}$ screen, 
Millipore), before passing via the cannula to the aorta. It was then recycled from the recording chamber back to the reservoir. The flow was generated with a peristaltic pump (Watson-Marlow 505D, Falmouth, Cornwall, UK) at a rate that was gradually increased from approximately 10 to $30 \mathrm{ml} / \mathrm{min}$, over about $1 \mathrm{~min}$. The perfusion pressure was monitored via the second lumen of the canula. Correct perfusion was confirmed by the observation of liver blanching and the brisk filling of the skull cavity. Once perfusate flow was initiated the heart resumed beating and rhythmic respiratory muscle contractions were seen within minutes, as perfusion pressure reached 50-60 $\mathrm{mmHg}$, signaling the return of brainstem function.

The preparation was not paralyzed with a muscle relaxant (except where specified) to retain EUS function and thus continence. As a consequence the preparation displayed respiratory movement and reflex withdrawal to tail/hindpaw pinch, which was used as a viability check throughout the experiments.

\section{PHRENIC NERVE RECORDING}

A glass suction electrode (tip diameter 250-350 $\mu \mathrm{m}$ ) held in a micromanipulator was used to record activity from the phrenic nerve. These electrodes were pulled from borosilicate glass capillary tubes (Harvard Apparatus; GC150TF-10). Signals were AC amplified and band pass filtered ( $50 \mathrm{~Hz}$ to $3 \mathrm{kHz}$ ). Rhythmic, ramping phrenic nerve activity, indicative of an eupnoeic pattern, gave a continuous physiological index of brainstem viability (Paton, 1996). The electrocardiogram (ECG) was also visible on the phrenic nerve recording and heart rate could be derived from the ECG, either online or offline, by triggering from the $\mathrm{R}$ wave.

During the initial stages of the experiment the preparation usually needed some "tuning" to maintain a eupnoeic pattern of respiration. This typically involved fine adjustment of flow from the pump, in combination with either vasopressor or peripheral chemoreflex activation. If the perfusion pressure was below $60 \mathrm{mmHg}$, the addition of vasopressin to the reservoir (final concentration $400 \mathrm{pM}$ ) elicited vasoconstriction and increased perfusion pressure. Once eupnoea was established, further addition of vasopressin during the course of the experiment (up to $4 \mathrm{~h}$ ) was not normally required. If phrenic activity was weak, a bolus arterial administration of sodium cyanide $(\mathrm{NaCN} ; 50 \mu \mathrm{l}$ of $0.03 \%)$ was injected into the perfusion line to stimulate peripheral chemoreceptors. This normally resulted in bradycardia and hyperpnoeic responses, following which phrenic activity would often be stabilized into a lasting eupnoeic pattern. The sodium cyanide was used sparingly, as repeated doses caused activation of the EUS, which may be a direct effect, or reflect altered central neural excitability following peripheral chemoreflex activation.

Once a eupnoeic pattern of phrenic activity was established, brainstem function was further assessed by monitoring the cardiorespiratory responses to afferent stimulation including activation of peripheral chemoreceptors ( $\mathrm{NaCN}$, as above), arterial baroreceptors (by increasing perfusate flow), trigeminal afferents (cold saline to snout to evoke diving response), and responses to noxious pinch of hindpaw or the tail. Preparations were considered to be non-viable when these reflex responses were lost. Indeed, their absence correlated with an inability to evoke a void.

\section{LOWER URINARY TRACT RECORDINGS}

The pubic symphysis was cut in the midline to access the EUS. To record EUS-EMG, a glass suction electrode (tip diameter $200 \mu \mathrm{m}$ ) was placed on the proximal sphincter, slightly lateral to the midline directly below the bladder neck under direct visual control. Suction was applied to draw a section of the sphincter into the recording electrode. A reference electrode was used to improve the signal: noise ratio and reduce ECG artifacts. The reference $\mathrm{AgCl}$ wire electrode was fixed to the outside of the glass capillary, with its free end positioned in close proximity to the glass suction electrode tip, either on an adjacent segment of the EUS or suspended in the fluid surrounding the EUS.

A 30G needle was inserted into the bladder dome for pressure monitoring and fluid infusion. This was connected via fluid-filled tubing and a three-way tap to a pressure transducer and a syringe pump allowing infusion of fluid (Kent Scientific). During insertion of the needle into the bladder dome, which required some handling of the bladder using blunt forceps, a momentary increase in EUS-EMG activity indicated intact bladder-EUS coordination. A video camera fitted to the binocular microscope allowed synchronous monitoring of bladder contractions and the precise timing of fluid ejection from the urethra (monitored against a contrasting background sheet).

The left bladder afferent nerve bundle, consisting of three to four branches exiting at the bladder neck was tracked and accessed after the major pelvic ganglion, where it became the pelvic nerve. This was approximately $2 \mathrm{~mm}$ away from the bladder neck. The nerve was dissected free from surrounding connective tissue and cut proximal to the ganglion for recording. A fine bipolar suction electrode $(\sim 50 \mu \mathrm{m})$ was used to record nerve activity during filling and voiding (Figure 1; inset).

\section{STIMULATION METHODS}

The bladder was filled by intermittent infusion with saline typically at a flow rate of $30 \mu \mathrm{l} / \mathrm{min}$. In experiments examining the voiding response to differential rates of filling (15-175 $\mu \mathrm{l} / \mathrm{min})$, both ureters were cut and ligated to stop natural bladder filling with urine from the kidneys. To stimulate bladder afferents, capsaicin solution $(100 \mu \mathrm{M})$ was infused into the bladder to trigger a single void before being washed out to trigger further voids. Mechanical pinch stimuli were applied to the hind limbs, tail, or the bladder, to assess the impact of sensory inputs on EUS activity. The distal urethra/ vulva was stimulated gently using a cotton swab. In some experiments, vecuronium bromide (topical, $2 \mu \mathrm{g} / \mathrm{ml} 10 \mu \mathrm{l}$; perfusate, $2 \mu \mathrm{g}$ / $\mathrm{ml}, 200 \mu \mathrm{l}$ ) was used to block the activity of the EUS. To test the effect of ganglion blockade on filling and voiding, hexamethonium (100-330 $\mu \mathrm{M}$; (Chizh et al., 1997)) was systemically administered via the perfusate.

\section{SEQUENTIAL HINDBRAIN TRANSECTIONS}

To confirm the involvement of hindbrain structures in the micturition reflex, a series of acute coronal brain transections were performed once the preparation was established and the voiding cycle had been elicited (see Smith et al., 2007). In these experiments, the posterior fossa was exposed and the cerebellum was removed during the cold dissection, before the preparation was perfused $(n=3)$. This allowed access to the midbrain, pons, and medullary 
regions during the experiment. In control experiments, the cerebellum was removed acutely, by suction aspiration, during continuous filling and voiding $(n=2)$. Hindbrain transections were then made at the predefined dorsal surface landmarks - between superior and inferior colliculi, and between rostral pons and caudal midbrain. At the completion of the protocol, the transected brain sections were fixed in situ with formaldehyde 10\% in PBS, before being sectioned parasagittally and stained using neutral red to identify nuclei.

\section{TIPS FOR SUCCESS}

During the development of this preparation, it was observed that the administration of heparin (i.p.) before beginning the surgery minimized the development of blood clots during the surgery. This allowed the perfusate to adequately reach the vasculature of the lower half of the preparation, specifically the lower spinal cord, the bladder and EUS. When heparin was administered during surgery or directly into the perfusate, it appeared to be less effective in preventing clotting which presumably caused patchy perfusion, resulting in inconsistent functional voiding responses.

The perfusion pressure required for robust eupnoeic activity and characteristic functional cardiorespiratory afferent-evoked responses (e.g., peripheral chemoreceptor reflex) was approximately $50 \mathrm{mmHg}$. However, at this perfusion pressure, coordinated bladder-EUS control was often lacking (along with reflex responses to noxious hindpaw and tail pinch). Bladder and sphincter coordinated control was achievable when perfusion pressure reached approximately $65-70 \mathrm{mmHg}$, likely reflecting the need to perfuse the distal segments of the spinal cord adequately. Anaesthetized rats of identical age show a similar arterial pressure (Kasparov and Paton, 1997). This was achieved by care to avoid opening the arterial tree during surgical preparation, administration of vasopressin, and judicious increments in perfusate flow to optimally tune the preparation.

\section{DATA ANALYSIS}

Perfusion pressure, phrenic nerve activity, ECG, bladder pressure, EUS-EMG activity, and bladder afferent nerve activity were recorded using custom built AC amplifiers and transducers (built by Mr. Jeff Croker, University of Bristol), and collected using a CED micro1401 A-D interface (CED, Cambridge Electronic Design, Cambridge, UK) to a computer running Spike2 software (CED). Analysis was conducted offline, using the Spike2 program and Prism 5.0. All values are expressed as the mean \pm standard error of mean or median (25-75 percentile) and $n$ is the number of preparations.

\section{DRUGS AND SOLUTIONS}

The composition of Ringer's was $\mathrm{NaCl}(125 \mathrm{mM})$ : $\mathrm{NaHCO}_{3}$ (24 mM), $\mathrm{KCl}(3 \mathrm{mM}), \mathrm{CaCl}_{2}(2.5 \mathrm{mM}), \mathrm{MgSO}_{4}(1.25 \mathrm{mM}), \mathrm{KH}_{2} \mathrm{PO}_{4}$ (1.25 mM); pH 7.35-7.4 after carbogenation. Ficoll-70 (1.25\%) was added as an oncotic agent. Arginine vasopressin (400 pM) was added to the perfusate. Stock solution of capsaicin $(10 \mathrm{mM})$ was made in 10\% ethanol, 10\% 2-hydroxypropyl-beta-cyclodextrin (HBC solvent) and $80 \%$ normal saline. Final working concentration of capsaicin $(100 \mu \mathrm{M})$ was made by diluting the stock in saline. All salts and drugs were from Sigma unless otherwise stated.

\section{RESULTS PREPARATION VIABILITY}

Once tuned, this preparation exhibits a robust eupnoeic pattern of phrenic activity that remains stable for periods up to $4 \mathrm{~h}$. The augmenting pattern of phrenic activity is indicative of good brainstem function, as reported previously (Paton, 1996; Pickering and Paton, 2006). It also shows strong cardiorespiratory coupling that manifests in both heart rate variability (respiratory sinus arrhythmia) and fluctuations in perfusion pressure (Figure 2A, and see Pickering and Paton, 2006). In the absence of muscle relaxant the preparation showed respiratory movements of the thoracic cage and upper airway muscles. Spinal reflexes were intact as assessed by motor responses to hind limb/tail pinch.

\section{CHARACTERISTICS OF NATURAL AND FLUID INFUSION EVOKED VOIDS}

Functional bladder and EUS neural coordination was clearly demonstrable in the preparation with both natural and infusion evoked voids (Figures 2 and 3). Natural voids were seen to occur in most preparations as fluid passing from the kidneys via the ureters filled the bladder. During natural filling, it was noted that the ureters displayed waves of peristalsis that propelled urine to the bladder (see Videos S1 and S2 in Supplementary Material). These peristaltic waves were observed as being initiated at the renal pelvis, and propagated caudally, toward the bladder. As fluid entered the bladder, the wave appeared to be propagated into the bladder itself, which displayed a spontaneous non-micturition contraction (NMC). The peristaltic waves from each kidney appeared to alternate, such that both kidneys did not pass urine to the bladder simultaneously, but rather in an inter-leaved fashion.

Both natural and infusion evoked voids had similar characteristics with voiding threshold pressure being $22 \pm 0.49 \mathrm{mmHg}$ $(n=10)$ and $23 \pm 1.0 \mathrm{mmHg}(n=12)$, respectively and a complete evacuation of bladder contents, as evidenced by direct visualization of the empty contracted bladder. In both types of voids, a gradual intra-luminal pressure increase was accompanied by a corresponding increase in tonic EUS activity, to keep the sphincter closed and maintain continence. At a threshold volume, measured from evoked voids only (median $23 \mu \mathrm{l}(21-62), n=12$; both ureters had been disconnected from the bladder to stop spontaneous filling), there was a coordinated contraction of the detrusor muscle (Figure 2; see Videos S1 and S2 in Supplementary Material) accompanied by a spike-like intra-luminal bladder pressure increase followed by ejection of urine from the urethra. In a series of initial experiments, collecting the volume evacuated from the urethra, it was confirmed that voiding was complete $(n=5)$. During the void the bladder pressure remained elevated at a plateau level with small, high frequency pressure oscillations (amplitude: $0.6 \pm 0.4 \mathrm{mmHg}$ ) that were generated by bursting activity of the EUS that was visually observed to intermittently narrow the urethral lumen (Figure 2B and inset), which is a characteristic of the normal rat voiding pattern (Maggi et al., 1986). The subsequent post-void pressure increase occurred as the bladder remained contracted whilst the EUS ceased bursting and resumed tonic firing.

The frequency of the EUS bursting activity was consistent between preparations, with an initial low frequency $(4.7 \pm 0.2 \mathrm{~Hz})$ that progressively increased during the void to a maximum $(6.9 \pm 0.4 \mathrm{~Hz}, P=0.0025)$, before a sudden cessation in bursting 


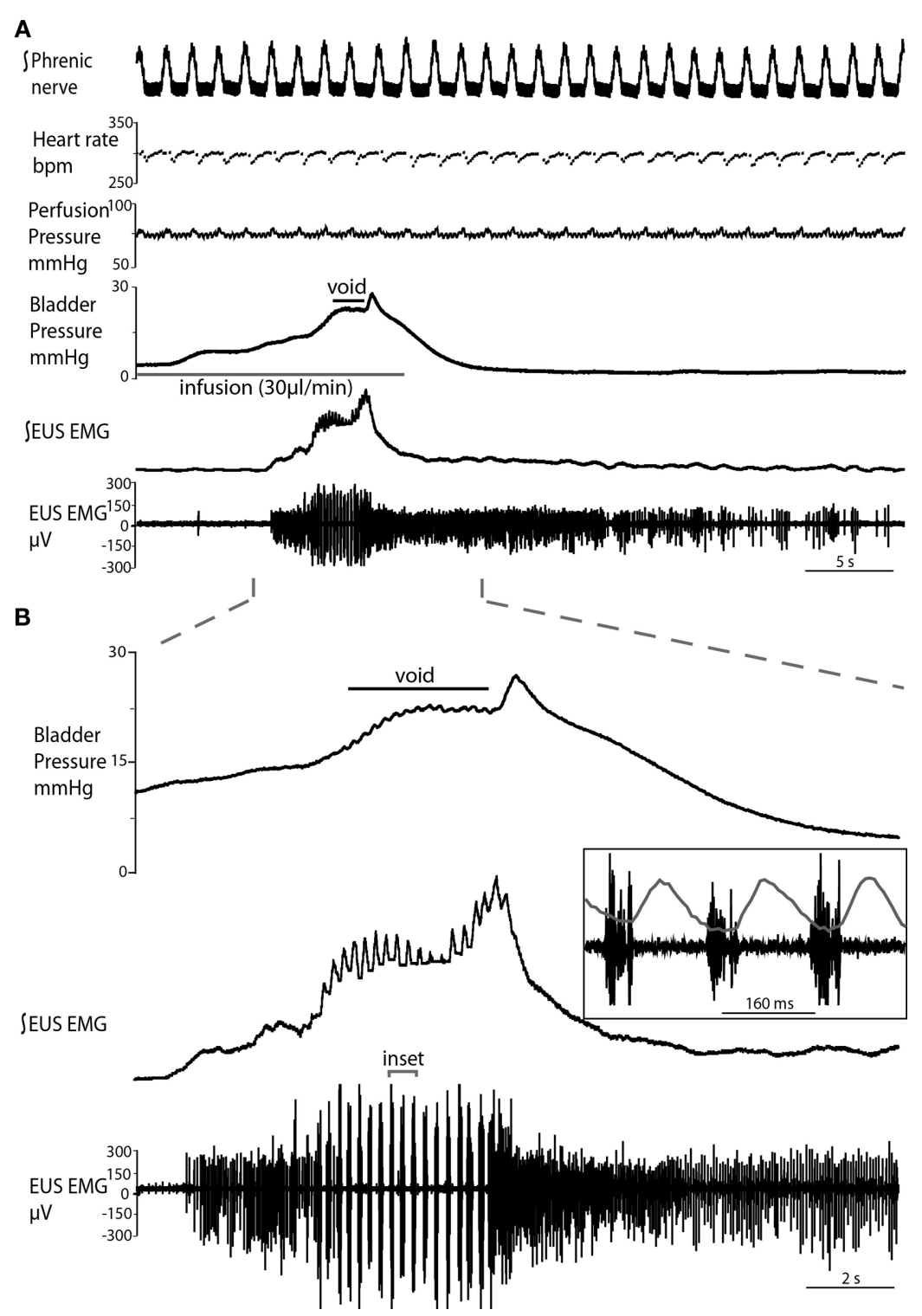

FIGURE 2 |Typical evoked micturition response. (A) As the bladder is filled there is a gradual rise in pressure and a tonic increase in activity of the EUSEMG. This pressure rise triggers a void with a generalized bladder contraction, a series of bursts on the EUS-EMG trace (mirrored by small oscillations in intravesical pressure) and the ejection of urine. Note at the end of the void as the tonic sphincter activity returns to baseline and the sphincter closes, the still contracting bladder generates a spike of pressure as it contracts isovolumetrically (also refer to Videos S1 and S2 in Supplementary Material)
(B) Expanded time scale showing increase in EUS activity during filling, followed by discrete bursting activity during voiding (inset: three individual bursts), in time with bladder pressure oscillations, where each burst is followed by a mini pressure rise (superimposed on inset), characteristic of the rat voiding pattern. Note in (A), the preparation also shows a eupnoeic pattern of phrenic nerve discharge with respiratory sinus arrhythmia seen in the heart rate trace consistent with intact brainstem-autonomic coupling yet none of these variables (nor perfusion pressure) are altered during the micturition reflex. activity and a return to tonic discharge. The amplitude of the EUS bursts was highest at the proximal EUS and decreased distally, as previously reported (Lehtoranta et al., 2006). Thus, all EUS-EMG measurements in the present study were taken at the proximal EUS. Gentle stimulation of the perineum using a cotton swab resulted in increased EUS tonic activity, regardless of bladder volume, causing the closure of the urethra, and indicating intact afferent function.
When the void was complete the bladder pressure returned to baseline. However, toward the end of the experiment, when brainstem control had started to deteriorate (e.g., non-eupnoeic phrenic pattern and cardiorespiratory reflex-evoked responses), incomplete voiding was observed. This reflects the requirement for intact brainstem function to coordinate the neural control of the lower urinary tract (as assessed from phrenic activity and cardiorespiratory reflex-evoked responses). 


\section{VOIDING RESPONSES AT DIFFERENT RATES OF BLADDER FILLING}

Variable urine infusion rates (from 15 to $175 \mu$ per minute) were used to examine the characteristics of the filling/voiding response $(n=4)$. As expected at low infusion rates $(15-30 \mu \mathrm{l} / \mathrm{min})$ it took longer before a void was triggered. These slow fills were also associated with more NMCs, which were accompanied with synchronous tonic EUS-EMG activity (Figure 4A). The bladder pressure at which voiding occurred was independent of infusion rates

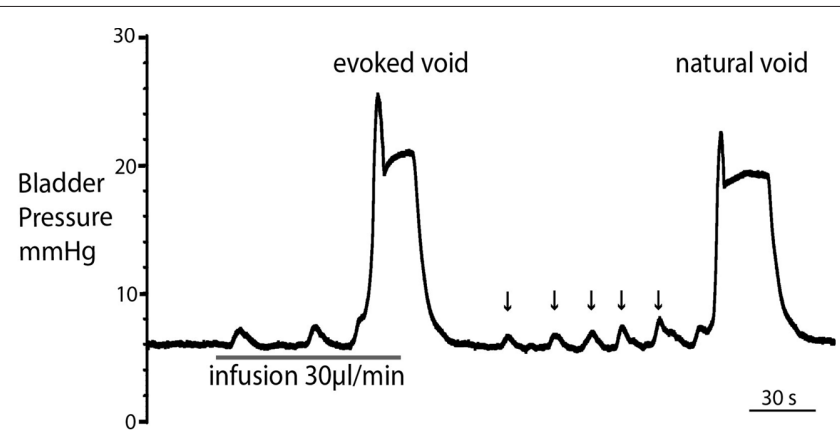

FIGURE 3 | Comparison of filling evoked and natural voiding responses. Natural voids occurred in most preparations as fluid from the kidneys filled the bladder. These were of qualitatively similar to filling evoked voids, with no significant differences in pressure trajectory or voiding threshold. Note also the presence of small, spontaneous non-micturition contractions (NMCs; arrowed)
(26.6 $\pm 0.3 \mathrm{mmHg} ; n=14$, Figure 4B). However, at higher infusion rates a greater volume of fluid was administered into the bladder before voiding was triggered $(P<0.0001$; Figure 4C).

\section{NON-MICTURITION CONTRACTIONS}

Spontaneous NMCs were seen as asymmetrical bladder wall movements in most preparations, even when the bladder volume was low (e.g., immediately post-void or when infusion was stopped). The NMCs had a frequency of $1.4 \pm 0.9$ per minute and intra-luminal pressure increase of $1.4 \pm 0.3 \mathrm{mmHg}(n=12$; Figure $5 \mathrm{~A})$. The NMCs were associated with phasic increases in EUS-EMG activity that presumably maintained continence. During active filling, as bladder volume increased, the amplitude of NMCs also increased (although the basal bladder pressure remained low), until void (Figure 5B). When brainstem function had deteriorated, marked by weakening phrenic nerve activity and loss of voiding, NMC amplitude increased significantly [5.8 $\pm 0.4 \mathrm{mmHg} ;(n=12)$ paired $t$-test $P<0.0001$; Figure 5C] but not frequency. In addition, changes were seen in the pressure waveform of NMCs after loss of brainstem control where biphasic waves were commonly seen (Figure 5C). Associated EUS activity was still present, but leakage of small volumes of urine was frequently seen but this was not a coordinated void.

\section{BLOCKADE OF THE EUS}

The topical application of a neuromuscular blocking agent, vecuronium bromide $(2 \mu \mathrm{g} / \mathrm{ml} ; 10 \mu \mathrm{l})$, to the EUS caused a decrease in EUS-EMG activity within $2 \mathrm{~min}$. In particular, there were fewer

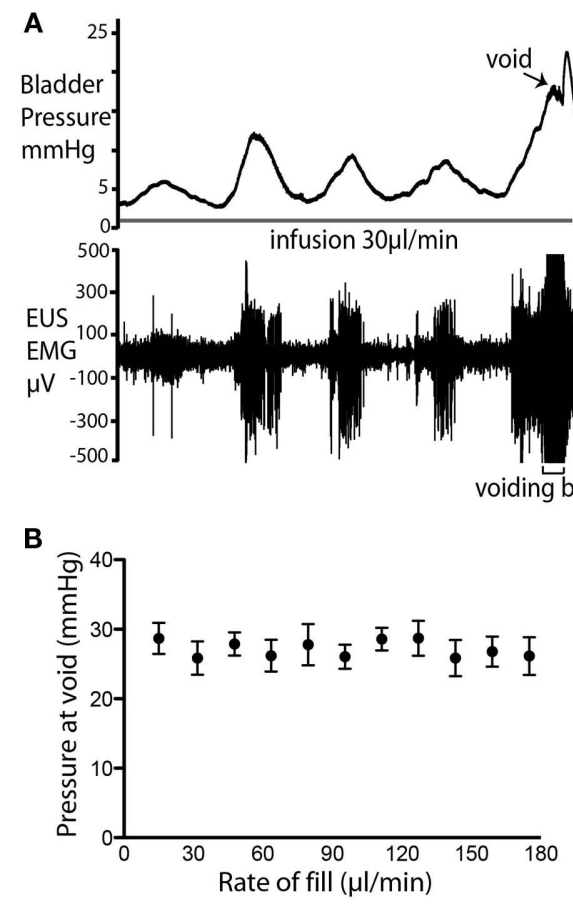

FIGURE 4 | Rate of fluid infusion and bladder pressure at void. (A) Example voids showing that at slower infusion rates (e.g., $30 \mu \mathrm{l} / \mathrm{min}$ ) it took longer for a void to be triggered and a larger number of NMCs were observed before the void. Note also the EMG activity that accompanied each NMC. At higher infusion rates,
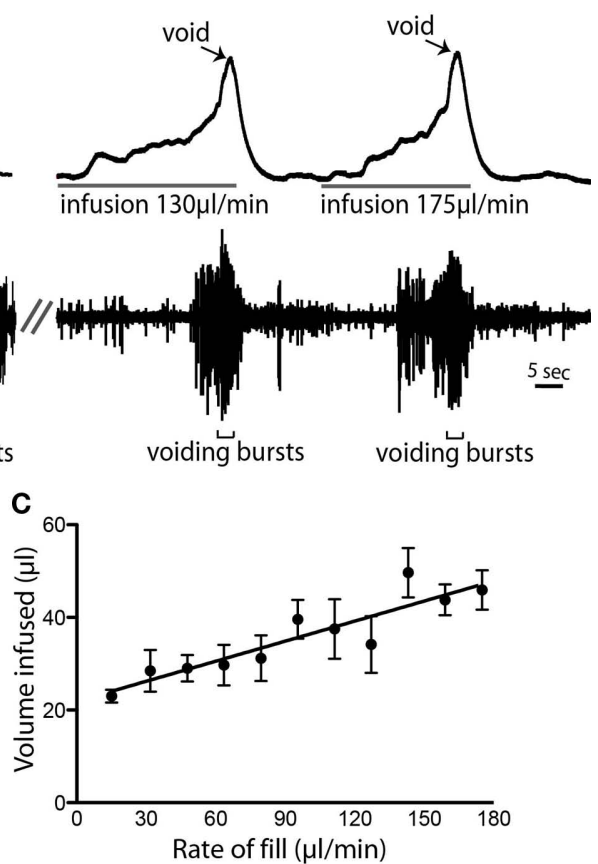

fewer NMCs occurred before the void. (B) The rate of fluid infusion did not significantly alter the bladder pressure at which the void occurred (C) A statistically significant linear relationship ( $P<0.0001 ; R^{2}=0.83 ; n=4$ ) was observed between infusion rate and the volume infused into the bladder before voiding was triggered. 


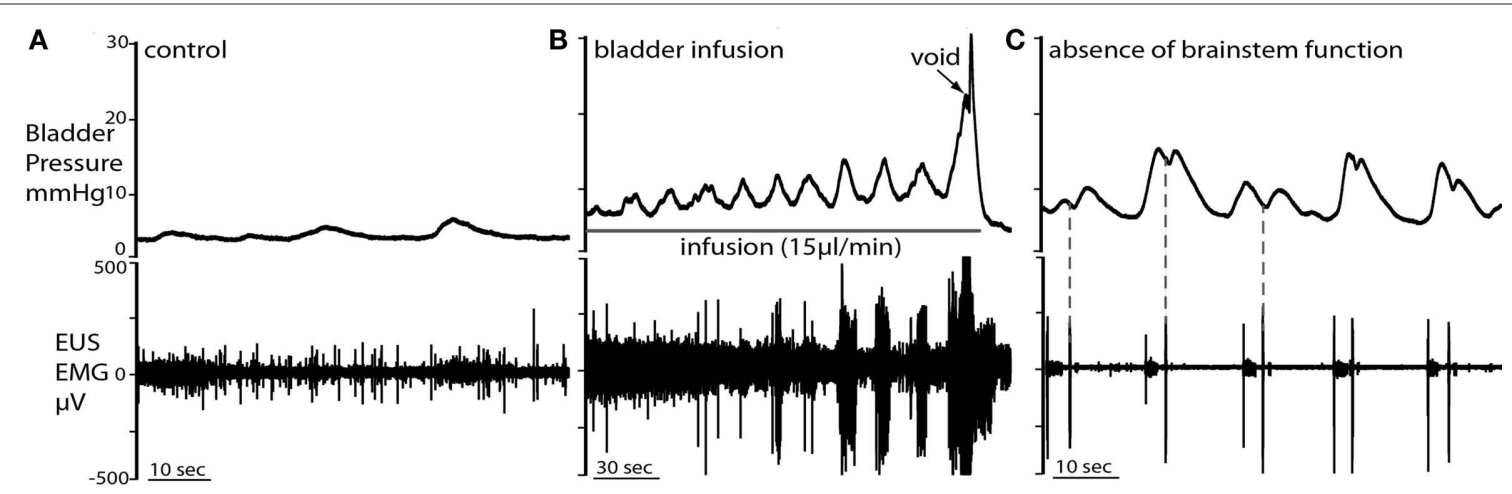

FIGURE 5 | Non-micturition contractions (NMCs). (A) Low amplitude NMCs occurred under basal conditions, when bladder volume was low. Each NMC was accompanied by tonic firing of the EUS. (B) During fluid infusion in the same preparation, NMCs became larger in amplitude with gradual bladder distension until voiding was triggered. (C) When brainstem control had deteriorated (as indicated by a loss of phrenic activity and voiding), the NMCs became biphasic and their amplitude significantly increased. In the first contractile phase of each NMC, tonic EUS firing was observed. The subsequent single burst of the EUS was followed by a second pressure oscillation (dotted line) and they could now be associated with leakage of fluid. and lower amplitude bursts during voids. A second application completely abolished EUS-EMG activity and further filling caused passive leakage of fluid, rendering the bladder incontinent (Figures 6Ai-iii; $n=4$ ). The local application of muscle relaxant did not attenuate the ongoing respiratory muscle activity seen in the thorax/neck indicating that the effects seen were local and not due to a systemic action.

Inclusion of the ganglion blocker, hexamethonium (100$330 \mu \mathrm{M}$ ), in the perfusate completely blocked the cardiovascular responses to peripheral chemoreflex activation (bradycardia and pressor effect) without changing the phrenic activation, indicating that the sympathetic and parasympathetic outflows were blocked. This autonomic ganglion blockade altered bladder pressure characteristics (Figures 6Bi-iii; $n=4$ ) with a decrease in voiding pressure, although the EUS bursting activity during voiding remained unchanged. After a further 2 min of ganglion block, the baseline intravesical pressure $(3.7 \pm 0.8 \mathrm{mmHg})$ increased by $116 \%$ and the NMC amplitude increased by $86 \%$ (Figure 6iii). During this period of ganglion block the EUS-EMG activity increased during each NMC to maintain continence. Voiding was still intact, but was incomplete and occurred at a lower voiding pressure with a loss of the post-void isovolumetric contraction.

\section{INTRAVESICAL CAPSAICIN}

Infusion of capsaicin $(100 \mu \mathrm{M})$ into the bladder produced a twophase response $(n=4)$. In the first phase, bladder filling with capsaicin solution caused an initial voiding response (Figure 7C), which showed a similar pressure trajectory to the control response (Figure 7A). However, several differences were noted; there was an increased level of tonic EUS activity (Figure 7C), particularly post-void, as capsaicin solution exited the bladder. Furthermore, voiding was incomplete and fluid retention occurred. In the second phase, this tonic EUS-EMG activity under basal resting conditions diminished and the EUS became silent, suggesting desensitization of bladder afferents. At this point filling challenges produced elevated intra-luminal pressure (by almost $2 \times$ fold to $40 \pm 3 \mathrm{mmHg}$; $P=0.0008$; Figure 7E) compared with control. As bladder pressure rose above approximately $20 \mathrm{mmHg}$ (previously the pressure threshold for voiding) it triggered an increase in EUS-EMG activity. At this maximum pressure irregular EUS bursting and brief pulses of fluid ejection occurred. This pattern of EUS bursting was atypical (with sporadic periods of silence interspersed on a tonic background) and this did not produce a normal voiding response. However the intermittent bursting allowed the bladder to sporadically release urine, eventually bringing the intra-luminal pressure back to baseline. The subsequent repeated infusion of saline failed to recover the normal voiding response.

Immediately after capsaicin infusion, large amplitude NMCs $(14.5 \pm 0.8 \mathrm{mmHg}$ in amplitude) compared with control $(2.3 \pm 0.4 \mathrm{mmHg}$ in amplitude, Figure $7 \mathbf{B})$, and associated EUS activity were observed (Figure 7D; $P=0.0001$ ). In phase 2, the NMCs decreased in amplitude and the basal level of EUS-EMG activity decreased (Figure 7F). Sporadic bursting responses, with associated incomplete bladder evacuation, remained until the completion of the experiment (up to $1 \mathrm{~h}$ post capsaicin infusion).

No changes in heart rate, perfusion pressure, or phrenic nerve activity were observed when capsaicin was administered into the bladder. Upon voiding of capsaicin solution, movement of hind limbs was observed, as solution exited and thus, stimulated the distal urethra and perineum.

\section{BLADDER AFFERENT AND PUDENDAL MOTOR NERVE RECORDINGS}

The right pelvic nerve was accessed proximal to the major pelvic ganglion. Afferent recordings showed a progressive ramping rise in activity during filling which followed the increase in bladder pressure $(n=4$; Figures $\mathbf{8 A}, \mathbf{B})$. Peak afferent activity was reached when EUS-EMG firing began to ramp (dotted line in Figure 8A), but plateaued before voiding began, potentially suggesting adaptation. This was followed by a period of bursting activity during the void, which was noted to be related to the EUS-EMG bursting but was in anti-phase. Post-void oscillations in afferent activity were also seen, as the bladder pressure returned to baseline. Pelvic afferent nerve activity was also triggered by changes in bladder pressure with each NMC, which was in phase with simultaneously recorded EUS-EMG activity. Systemic application of vecuronium 

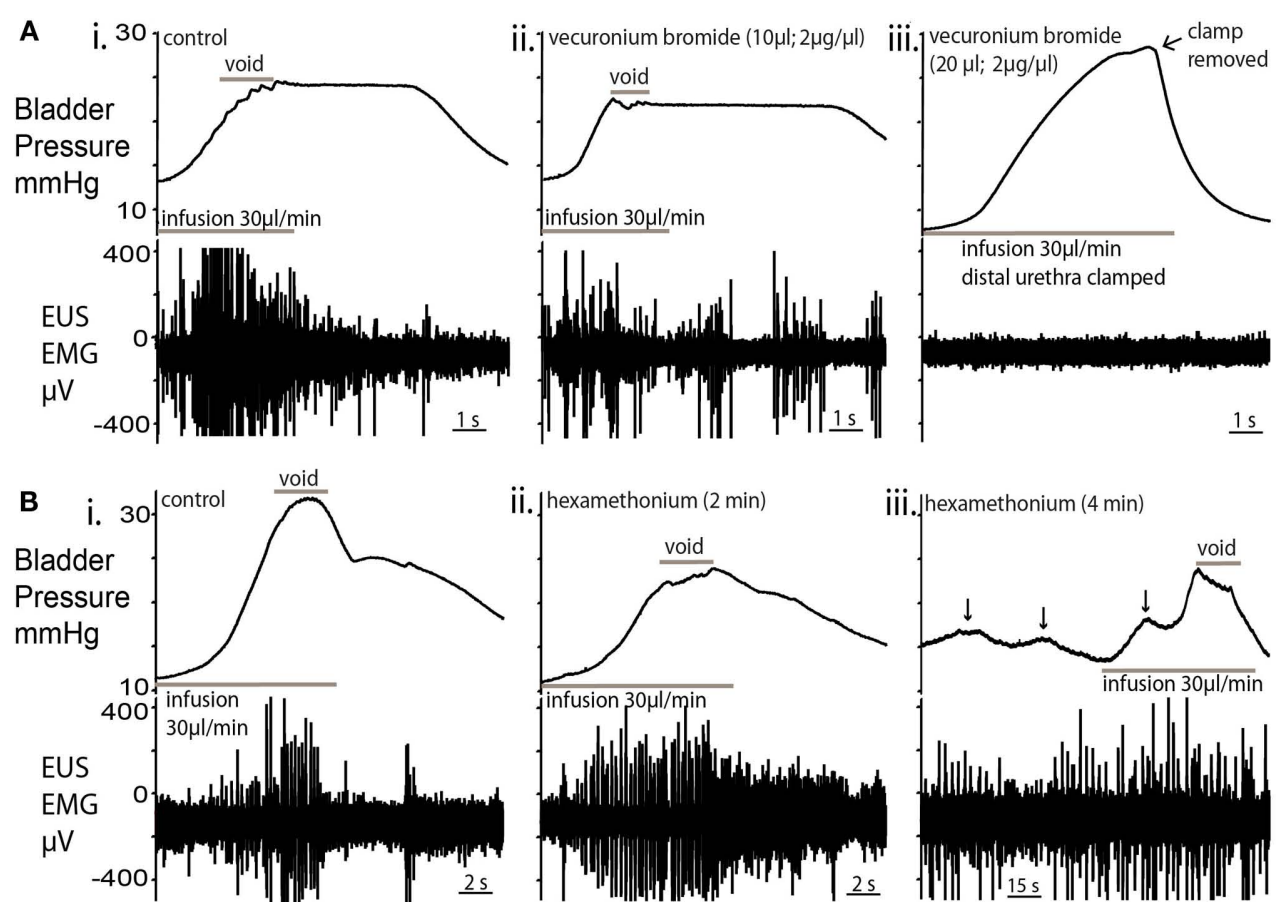

FIGURE 6 | Effect of nicotinic receptor blockade on micturition. (A) Topical vecuronium bromide (a competitive antagonist of neuromuscular transmission) applied to the EUS $(10 \mu \mathrm{l} ; 2 \mu \mathrm{g} / \mu \mathrm{l})$; (ii), caused a decrease in EUS-EMG activity within 2 min but voiding still occurred, albeit at a lower pressure [compared to control; (i)]. In particular, during the void, fewer and lower amplitude EUS-EMG bursts were observed. (iii) A second application of vecuronium completely abolished EUS-EMG activity and further infusion caused passive leakage of urine. In order to test the EUS activity, the distal urethra was clamped to allow bladder pressure to increase. No EUS activity was evoked. This block was maintained for the following 30 min without sign of recovery. (B) Application of the ganglion blocker hexamethonium [330 $\mu \mathrm{M}$; (ii)] to the perfusate blocked the cardiovascular responses to peripheral chemoreflex activation indicating that the sympathetic and parasympathetic outflows were blocked. There was an initial decrease in the voiding pressure, although the characteristic pressure trajectory and EUS bursting activity remained. (iii) After a further 2 min, NMCs became larger (arrowed) in amplitude, with associated EUS activity to maintain continence. Voiding was present, but altered, with low voiding pressure and high baseline intravesical pressures. bromide abolished the EUS-EMG activity (as well as all gross motor movements), however afferent nerve firing continued to be evoked by bladder distension (following fluid infusion with the urethra clamped) and also in response to NMCs although these now caused leakage of urine indicating the importance of the EUS activity in the maintenance of continence during these small pressure fluctuations (Figures 8C,D).

\section{BRAIN TRANSECTION}

We tested the effect of a series of acute brain transections (from rostral to caudal) on the neural control of the bladder once the preparation had been established. Initial control experiments showed that acute cerebellectomy had no effect on filling and voiding responses, with similar voiding pressure and infused volume $(n=3$; data not shown). Similarly sections at the level of the superior colliculus were without effect. However the next transection at a mid-collicular level consistently resulted in the loss of coordinated voiding (Figure 9; $n=3$ ). Subsequent histological analysis showed that this section disrupted the rostral PAG in the midbrain but was still some distance rostral to the PMC. Following this section filling responses remained so that bladder pressure increased as fluid was infused, together with associated increase in EUS-EMG activity. At threshold volume, some leakage of fluid occurred, but no active void occurred. With each transection the phrenic activity became transiently apneustic however, once re-tuned, the preparation continued to display robust phrenic activity and normal cardiorespiratory reflex-evoked responses.

\section{DISCUSSION}

The primary purpose of this study was to develop and validate a novel in situ animal model of the control of micturition without the confounding effect of anaesthesia and with the advantages of an in vitro approach. The DAPR offers some advantages for the study of bladder autonomic control and allows the completion of complex experimental protocols in a relatively short time (Pickering and Paton, 2006). The preparation allowed excellent access to the EUS and afferent and efferent nerves for recordings under direct vision, allowing correlations to be made between bladder and EUS movement, voiding and the electrical activity in muscle and nerve. The influence of autonomic tone and the critical role of inputs from brainstem/midbrain centers was clearly demonstrable indicating that the preparation has utility for examination of these central and peripheral neural mechanisms. Further the approach allows straightforward drug application either systemically (at known concentrations), locally or intravesically and permits the use of substances that would be toxic to in vivo preparations. Importantly we were able to demonstrate the ability to "open the loop" of the micturition reflex recording 

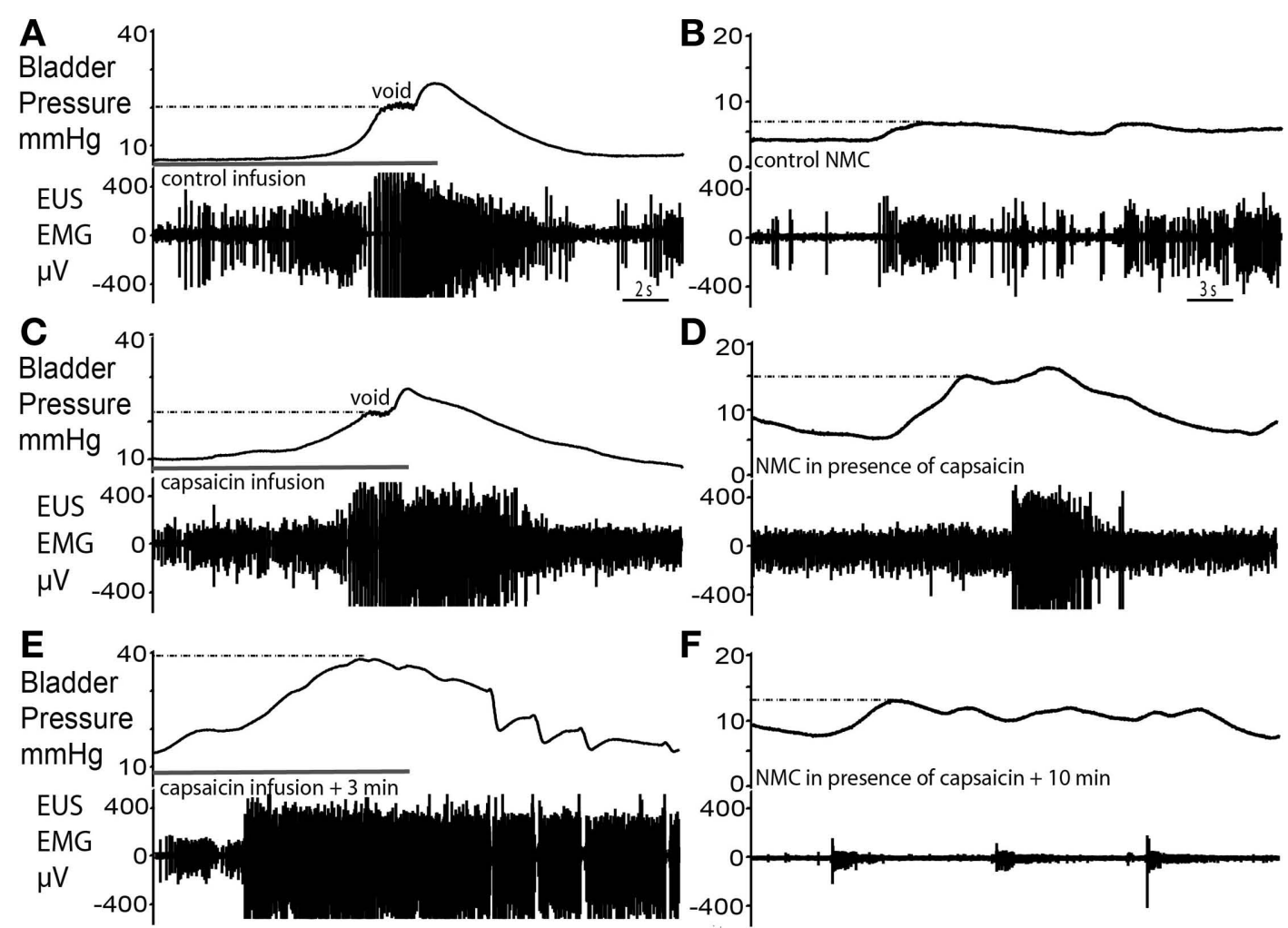

FIGURE 7 | Capsaicin sensitization-desensitization of bladder responses. (A,B) Control void and spontaneous NMCs. (C) Filling with capsaicin solution caused an initial voiding response of similar pressure characteristics as control. However, an increased level of tonic EUS activity particularly post-void was observed and voiding was incomplete. (D) At this time there was a marked increase in the amplitude of NMCs and they were accompanied by larger bursts of EMG activity. (E) Some 3 min after the application of capsaicin, intravesical infusion at the same rate continued for a longer period of time until almost twice the volume had been administered and intravesical pressure had increased twofold above that seen during the control void. This infusion triggered a striking increase in the level of EUS-EMG activity as bladder pressure rose above $20 \mathrm{mmHg}$, previously the pressure at which voiding occurred. However, small incomplete voiding episodes were seen to occur at the highest pressures accompanied by irregular EUS bursting. Normal voiding responses were non recoverable. (F) The subsequent NMCs resulted in synchronous low level tonic firing, as the EUS was markedly desensitized.
nerves/EMG and intervening to control bladder filling/volume while retaining intact end organ function and neural control; a situation analogous to what we have previously been able to do in similar preparations to examine cardiorespiratory control in a way that is difficult if not impossible in vivo (Boscan et al., 2002; Simms et al., 2007; Smith et al., 2007; Pickering et al., 2008; Abdala et al., 2009).

\section{RAT VOIDING CHARACTERISTICS}

Characteristic rat bladder filling (Maggi et al., 1986; Thor and de Groat, 2010) and voiding responses were evoked by the intravesical infusion of fluid, featuring a gradual increase in pressure, followed by coordinated contraction of the bladder producing spikes in intravesical pressure and voiding of urine. A final post-void isovolumetric pressure increase subsequently occurs, as the EUS returns to tonic activity while the bladder remains contracted. This micturition cycle also occurred naturally, as filtrate passing via the ureters from the kidneys (in peristaltically propelled boluses) filled the bladder.

By ligating the ureters to stop natural bladder filling, it was possible to examine the relationship between intravesical fluid infusion/ volume and voiding. The rate of fluid infusion did not affect the pressure at which the void was triggered, however at higher infusion rates the void was triggered at greater infused volumes. This suggests that the bladder compliance decreases with slow rates of infusion, perhaps as consequence of sympathetic withdrawal. This may indicate a time lag in sympathetic withdrawal, which is known to influence bladder compliance (Khadra et al., 1995).

The preparation allowed clear visualization of the entire length of the EUS making EMG recording straightforward. The pattern of EMG activity through the micturition cycle was as has previously been characterized for the female rat (Streng et al., 2004; Walters et al., 2006) with a graded increase in activity during filling, a switch to phasic bursting during the void and a tonic phase following cessation of voiding. EMG responses were recordable along the full length of the EUS, although the bursting activity seen during voiding was strongest at the proximal lateral EUS, as has been previously reported (Lehtoranta et al., 2006). Local application of vecuronium, a non-depolarizing muscle relaxant, caused a loss of EUS electrical activity and tone (and urinary continence), as expected for a voluntary muscle (Bennett et al., 1995) without blocking the movement seen in respiratory pump muscles nor those of the abdominal/pelvic wall confirming that the signal was recorded from the EUS. 

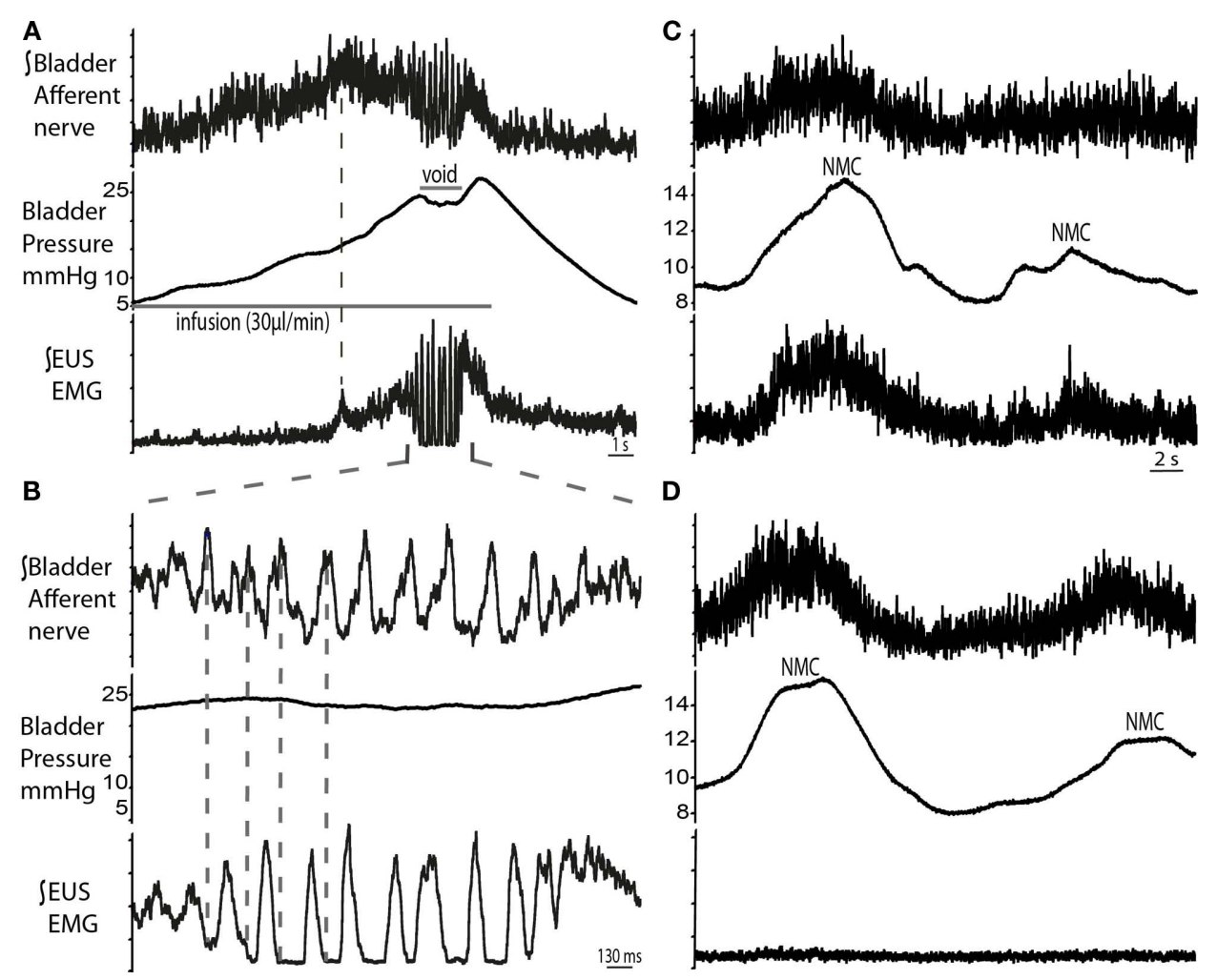

FIGURE 8 | Bladder pelvic nerve afferent activity during micturition cycle. (A) Bladder afferent nerve activity increased during filling, followed by a drop in activity before the void $(n=4)$. The ramping discharge of the afferent nerve was progressive and followed the increase in bladder pressure. At void, afferent nerve bursting was observed to be in anti-phase with the EUS bursting activity [expanded in (B)]. (C) Changes in bladder pressure during NMCs were associated with increase in both, EUS-EMG and afferent nerve discharge. To ascertain that this was a nerve response, muscle relaxant was applied (vecuronium dose $2 \mathrm{\mu g}$ / $\mathrm{ml}$, systemically) (D), which resulted in complete abolition of EUS activity, while afferent activity remained on the pelvic nerve in synchrony with the NMCs.
The simultaneous monitoring of EUS-EMG, bladder pressure and afferent activity in the pelvic nerve allowed us to identify a unique pattern of discharge on the nerve in anti-phase to the bursting activity on the EUS during the second phase of micturition. The amplitude of these afferent oscillations appeared disproportionately large compared to any synchronous change in bladder pressure and it seems likely to be related to flow in the proximal urethra which occurs between the bursting EUS contractions (Streng et al., 2004). We speculate that this bursting afferent feedback plays an important role in the generation of the EUS bursts that characterize the second phase of micturition in the rat.

\section{GANGLION BLOCKADE}

Application of the ganglion blocker hexamethonium changed the pressure characteristics of the bladder. The increased baseline and large amplitude NMCs are indicative of the loss of sympathetic drive, which is primarily involved in the storage phase of micturition, to increase bladder compliance (Clemens, 2010). The presence of normal autonomic tone suppresses these spontaneous pressure changes, permitting greater bladder compliance (Clemens, 2010). However, in the rabbit catheterized in vivo bladder preparation (Levin et al., 1986), hexamethonium application completely abolished this spontaneous activity, suggesting that NMC initiation (but not inhibition) is neuronal. Species-specific differences may explain these discordant findings.
During filling, continence was maintained via intact afferent signaling from the bladder and intact somatic motor somatic control on the EUS. Parasympathetic drive, primarily involved in voiding, maintains detrusor contraction during the void and even after the completion of the void, resulting in the isovolumetric contraction. In the present study, voiding was incomplete and occurred at lower threshold pressure after hexamethonium administration. There was also a loss of the end isovolumetric contraction. All of these results signal the loss of actively augmented detrusor contraction during the void, as a result of disruption of parasympathetic drive. However, the somatically controlled bursting of the EUS was able to permit an incomplete void.

Another documented action of hexamethonium is its antagonistic effect at nicotinic acetylcholine receptors on the skeletal muscle end plate (Tian et al., 1997) and it may thus be proposed to block EUS activity and abolish voiding. At concentrations between 100 and $330 \mu \mathrm{m}$ in the perfusate we saw no evidence for blockade of skeletal muscle movement nor on EUS-EMG although we did clearly document the presence of dense autonomic ganglion blockade. The in vivo systemic effect of hexamethonium on the bladder and EUS has not been extensively investigated, however its action at the neuromuscular junction is considered to be less potent than its actions as a ganglion blocker, which is line with its historical use the treatment of hypertension (Mathias, 1991). 


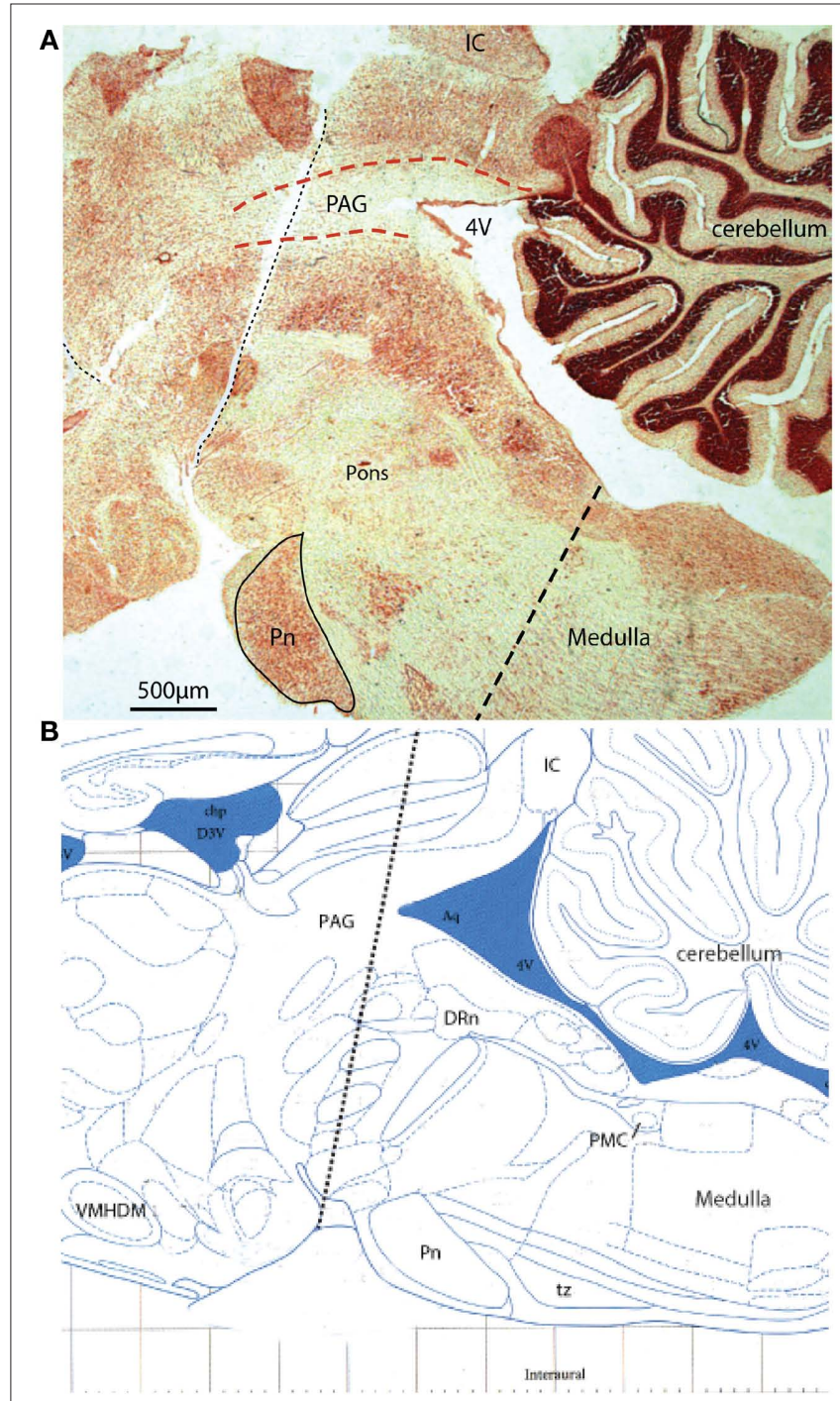

FIGURE 9 |Acute sequential hindbrain transections. (A) Histological parasagittal section $(0.18 \mathrm{~mm}$ lateral) through hindbrain showing the mid-collicular line of transection (Neutral red stained section) and (B) annotated schematic (Paxinos and Watson, 2007) of the acute brainstem transections during active filling and voiding. The initial transection dissociated the rostral tissues from the brainstem (e.g., hypothalamic nuclei) leaving the brainstem intact. Voiding remained unaffected by this intervention. The second transection (shown) effectively disrupted the rostral periaqueductal gray (PAG), and resulted in loss of coordinated voiding, although filling responses remained. DRn, dorsal raphe nucleus; IC, inferior colliculus, $\mathrm{Pn}$, pontine nucleus; tz, trapezoid body, VMHDM, ventromedial hypothalamic nucleus dorsomedial.

\section{NON-MICTURITION CONTRACTIONS}

NMCs were observed both under basal conditions (with the bladder relatively empty) and during fluid infusion into the bladder. These NMCs may represent an "assessment reflex" where the bladder continuously monitors its level of fullness (Finney et al., 2008). Indeed we were able to record simultaneously both the afferent signal in the pelvic nerve resulting from NMCs and the subsequent proportionate response from the EUS-EMG reflecting contraction which is clearly required to maintain continence (lost in the pres- ence of neuromuscular blockers) indicating that these contractions are both centrally monitored and acted upon as would be expected for an assessment reflex. The peristaltic propulsion of fluid from the kidneys via the ureters was observed to initiate an NMC. In addition, the coordinated alternating fashion of propelling urine (not previously reported to our knowledge) may suggest a neurogenic (rather than myogenic) component to the co-ordination of ureteric function, a topic of continuing debate (Kreulen et al., 1977; Santicioli and Maggi, 1998; Osman et al., 2009). Slower infusion rates were shown to induce a larger number of NMCs before the initiation of the void, and they were non-existent at higher infusion rates $(>110 \mu \mathrm{l} / \mathrm{min})$. It is possible that NMCs are inhibited by larger rates of change in bladder volume and afferent activity. Alternatively, a more likely explanation may be that the time taken for initiation of NMC is longer than the infusion rate. This may be consistent with the proposal that NMCs are triggered by the slow release of mediators from neuronal/non-neuronal cell types within the bladder (Kanai et al., 2007).

When brainstem control was compromised, the spontaneous NMCs observed became biphasic and had increased amplitude. This was also seen after autonomic ganglion blockade with hexamethonium (discussed above). This is in line with previous studies that have shown that spontaneous bladder activity in the rat is reliant on, and tonically inhibited by, input from the lumbo-sacral spinal cord via parasympathetic pathways (Maggi et al., 1986; Sugaya and de Groat, 2002). In the rat, the occurrence of NMCs has been reported to be age dependent - they are present and have high frequency and amplitude in younger bladders, declining in adult bladders, but re-emerging as high intensity NMCs in older bladders (Szigeti et al., 2005). It can be speculated that changes observed in aged bladders may be due to disruption in the bulbospinal control circuitry and/or loss of autonomic tone, similar to those seen in the present study, during loss of brainstem control.

\section{STIMULATION WITH CAPSAICIN}

Capsaicin is known to excite and then desensitize bladder C-fiber afferents (Maggi et al., 1986; Maggi, 1991). In the present study, although the initial voiding response characteristics after capsaicin infusion were relatively unchanged from the control void (i.e., unchanged pressure trajectory and EUS bursting), two main differences were observed: presence of a residual volume and elevated tonic EUS activity, which are both in line with the initial excitatory effects of C-fiber activation. These afferents are of the high threshold type which are considered to respond to noxious stimuli, but not normally to distension (Habler et al., 1990). In the present study, it is likely that capsaicin infusion caused initial activation of C-fibers, resulting in the initiation of a voiding response (to eject the irritant substance). As fluid exited the bladder, urethral afferents were excited by the capsaicin, causing heightened activity of the sphincter, thus tightening of the bladder neck. This may have resulted in premature closure of the urethra and an incomplete void.

The desensitizing effect was evident within $10 \mathrm{~min}$ as the bladder pressure threshold for voiding increased twofold, before intermittent voiding contractions were observed. Indeed, the rationale for using capsaicin as a potential treatment for over active bladder is this increase in bladder capacity due to desensitized afferent C-fibers (Chancellor and de Groat, 1999). However, 
data in this regard is controversial as these C-fibers are considered by some to be silent in normal micturition (Habler et al., 1990; Andersson, 2002; Fowler, 2002). Treatment using capsaicin was thought to be beneficial for patients with detrusor overactivity where C-fiber involvement is enhanced in micturition (Chancellor and de Groat, 1999). However, capsaicin treatment has had a variable clinical success rate (Geirsson et al., 1995; Das et al., 1996; de Seze et al., 2004). A-delta fibers, which respond to normal distension, are also responsive to capsaicin (Chancellor and de Groat, 1999), which results in the inhibition of micturition in the rat for up to $5 \mathrm{~h}$ (Cheng et al., 1993). Thus, data from this study, showing the increase in pressure and loss of normal voiding after capsaicin infusion, adds further credibility to the theory that afferent fibers expressing TRPV1 are more than just receptors of noxious stimuli, but rather some at least appear to be involved in normal bladder function (Birder et al., 2002; Daly et al., 2007; Gevaert et al., 2007).

\section{BRAINSTEM CONTROL OF MICTURITION}

The assessment of the roles of brainstem nuclei in bladder control has been challenging for a number of reasons including the inaccessible nature of the brainstem regions involved and also the requirement for anaesthesia for these types of in vivo studies. Electrical stimulation of brainstem nuclei with simultaneous recordings from pelvic and postganglionic nerves in rats has previously indicated that the neural switching circuit controlling micturition is located in the rostral brainstem (Kuru, 1965; Noto et al., 1991). In addition, stimulation of the PAG in cats can evoke micturition (Taniguchi et al., 2002). In man, functional brain imaging studies have been undertaken although these tend to be focused upon higher centers as the brainstem is difficult to image (Blok et al., 1997; Athwal et al., 2001; Griffiths et al., 2005; Zhang et al., 2005; Kitta et al., 2006). In these studies, the neural mechanisms being investigated are influenced by confounding brain processes, including attention, expectation, decision, and emotion, which are highly variable and complicate interpretation of data (Mayer et al., 2006). In an investigation of the basic micturition reflex, imaging studies performed on anaesthetized rats showed the PAG to be activated during filling, and still further activated at threshold volume, while the PMC was inactive during filling but activated at threshold (Tai et al., 2009).

We took advantage of the non-anaesthetized in situ model to make systematic acute hindbrain transections from rostral to caudal to investigate the level at which the phases of micturition were eliminated (analogous to the approach we have taken to identify the components of the respiratory pattern generating network; Smith et al., 2007). These preliminary data confirmed the importance of the PAG for voiding, which ceased when the PAG was disrupted, although filling responses of the bladder and EUS remained intact. Indeed these filling responses were preserved with more caudal brainstem transections and in a spinal preparation (unpublished observations). These interventions emphasize that intact brainstem/midbrain function is required for micturition and that these structures are easily accessed and manipulated in this in situ preparation. This opens the gateway to deploy more discrete interventions to test the functional roles of the components of the bladder control network. We have previously made extensive use of the excellent access to brainstem structures to perform microinjection studies of agonists and antagonists (Boscan et al., 2001; Simms et al., 2006), to record cell populations both extracellularly and intracellularly (Paton et al., 2000) and even to make imaging studies using voltage sensitive dyes (Potts and Paton, 2006). These approaches are facilitated by direct vision of the surface of the brainstem, the good mechanical stability in the absence of a pulse wave and the lack of bleeding. We anticipate that the use of these types of recording approaches in the DAPR preparation will facilitate future explorations of the functional role of the brainstem control centers (i.e., PMC/PAG) in the initiation and regulation of voiding.

\section{TECHNIOUE DEVELOPMENT}

During initial development of the preparation filling and pressure monitoring was attempted via the ureters, where fine cannulae were inserted down the length of the ureter toward the bladder. However, when instrumenting the ureters when the preparation was established, there was an apparent effect on phrenic nerve activity. Each phrenic burst became shorter, and the ramping eupnoeic pattern was often lost. This may be a ureteric-evoked noxious response analogous to those accompanying ureteric stones (Ng et al., 2005). Robust eupnoeic phrenic activity was often difficult to re-establish, once lost. Furthermore it was difficult to traverse the ureto-bladder junction with the cannula preventing intravesical pressure recording so this approach was abandoned in favor of the more conventional pressure monitoring/infusion needle inserted into the bladder dome (Streng et al., 2004; Yoshida et al., 2010).

Gentle stimulation of the perineum using a cotton swab resulted in increased EUS tonic activity, regardless of bladder volume. A similar response was seen following insertion of a cannula into the distal urethra. Thus, cannulation of distal urethra was avoided, as subsequent bladder filling and voiding responses were likely to be affected, due to greater EUS firing than normal.

Nociceptive responses were elicited in the preparation as seen by tail/paw pinch reflex withdrawal responses as well as associated increases in heart rate and phrenic discharge. At the end of experiments, when brainstem function had deteriorated as evidenced by loss of reflex responses and a weak apneustic respiratory pattern, filling responses remained but voiding became first incomplete and then was not achievable, resulting in retention of urine. During acute spinal cord injury, the bladder is known to become areflexic, where it continues to fill but lacks the ability to switch to voiding (de Groat and Yoshimura, 2010). This suggests that deterioration of the preparation (typically after around $4 \mathrm{~h}$ of experimentation) likely occurs at brainstem level first, leaving spinal pathways intact, permitting filling, but not voiding, to continue.

Age related differences might also be the cause of urine retention in some of the preparations in the present study. It is believed that rats below the age of $\mathrm{P} 19$, require maternal perineal stimulation for complete bladder emptying (Kruse and De Groat, 1990). In the present study, approximately $10 \%$ of preparations (P20-P28) showed incomplete bladder emptying in the control situation. It is possible that the age group of animals used here are in a borderline developmental stage, where some continue to display characteristics of neonates. 


\section{ADVANTAGES AND LIMITATIONS OF THE PREPARATION}

We have shown that the DAPR is a viable and robust model for bladder autonomic studies. The preparation simplifies surgical access to pelvic nerves as well as the EUS in a bloodless field. The perfusion style preparation is advantageous allowing in vitro like control over the extracellular fluid and permits infusion of known concentrations of compounds either systemically (even of toxic substances that would be lethal in a conventional anaesthetized preparation (see Pickering et al., 2002)) or by intravesical or topical application. The use of this approach has already permitted novel insights to be gained as a consequence of the remarkable visual access to normally inaccessible territories (allowing both ureters to be visualized and observed to be contracting in an alternating fashion with these peristaltic waves continuing as bladder NMCs) and also by facilitating simultaneous recordings of nerves, muscles, and pressures in a non-anaesthetized voiding preparation (allowing the relationship between afferent pelvic nerve and EUS motor activity to be defined). Furthermore, the simultaneous recordings of phrenic nerve activity, heart rate, and perfusion pressure provides a measurement of cardiorespiratory responses during normal and pathological bladder function, which is an area that has been little researched. The comparative ease and speed with which such experiments can be performed is also a major advantage over more conventional in vivo approaches.

A potential drawback of the preparation was the use of young rats (P20-P28; weighing 40-90 g), which was primarily due to the difficulty in establishing adequate tissue perfusion in larger animals which limits preparation viability. However, this can be overcome by the use of adult mice (Paton, 1996). Technical limitations include the duration of viability (up to $4 \mathrm{~h}$ ), which can place time constraints on complex experimental protocols, however, this is counterbalanced by the initial relative speed of set up of the preparation which gives a good working window for experimentation. Furthermore, since retaining EUS function mandated that muscle relaxant could not be used, the preparation exhibited prominent respiratory and evoked reflex movements, which could make recording procedures challenging. The bloodless working field was advantageous as it allowed clarity of vision, however it also resulted in the loss of tissue contrast, which is particularly helpful when identifying small nerve branches.

In conclusion, this preparation facilitates recording and manipulation of the neural control of the bladder from the terminal afferent field through the spino-bulbo-spinal pathway and back to the motor outflow and end organ responses during the integrated micturition cycle. Electrophysiological recordings from the associated structures were straightforward and showed responses comparable to those previously documented from conventional in vivo experimentation. This model is ripe for extension to encompass bladder pathologies and use to assess the utility of potential new treatments.

\section{ACKNOWLEDGMENTS}

Astellas Pharma Inc. provided unrestricted grant funding for research. The authors are grateful to Cees Korstanje and Frank Perabo for their insights, encouragement and productive discussions. Julian F. R. Paton was in receipt of a Royal Society Wolfson Research Merit Award. Anthony E. Pickering is a Welcome Trust Senior Clinical Fellow.

\section{SUPPLEMENTARY MATERIAL}

The Supplementary Material for this article can be found online at http://www.frontiersin.org/Autonomic_Neuroscience/10.3389/ fnins.2011.00062/abstract

\section{REFERENCES}

Abdala, A. P., Rybak, I. A., Smith, J. C., and Paton, J. F. (2009). Abdominal expiratory activity in the rat brainstem-spinal cord in situ: patterns, origins and implications for respiratory rhythm generation. J. Physiol. 587, 3539-3559.

Andersson, K. E. (2002). Bladder activation: afferent mechanisms. Urology 59, 43-50.

Athwal, B. S., Berkley, K. J., Hussain, I., Brennan, A., Craggs, M., Sakakibara, R., Frackowiak, R. S., and Fowler, C. J. (2001). Brain responses to changes in bladder volume and urge to void in healthy men. Brain 124, 369-377.

Avelino, A., Cruz, C., Nagy, I., and Cruz, F. (2002).Vanilloid receptor 1 expression in the rat urinary tract. Neuroscience 109, 787-798.

Bennett, B. C., Kruse, M. N., Roppolo, J. R., Flood, H. D., Fraser, M., and de Groat, W.C. (1995). Neural control of urethral outlet activity in vivo: role of nitric oxide. J. Urol. 153, 2004-2009.

Birder, L.A., Nakamura, Y., Kiss, S., Nealen, M. L., Barrick, S., Kanai, A. J., Wang, E., Ruiz, G., De Groat, W.C., Apodaca, G., Watkins, S., and Caterina, M. J. (2002).
Altered urinary bladder function in mice lacking the vanilloid receptor TRPV1. Nat. Neurosci. 5, 856-860.

Blok, B. F., Willemsen, A. T., and Holstege, G. (1997). A PET study on brain control of micturition in humans. Brain 120(Pt 1), 111-121.

Boscan, P., Allen, A. M., and Paton, J. F. (2001). Baroreflex inhibition of cardiac sympathetic outflow is attenuated by angiotensin II in the nucleus of the solitary tract. Neuroscience 103, 153-160.

Boscan, P., Kasparov, S., and Paton, J. F. (2002). Somatic nociception activates NK1 receptors in the nucleus tractus solitarii to attenuate the baroreceptor cardiac reflex. Eur. J. Neurosci. 16, 907-920.

Chancellor, M. B., and de Groat, W. C. (1999). Intravesical capsaicin and resiniferatoxin therapy: spicing up the ways to treat the overactive bladder. $J$. Urol. 162, 3-11.

Cheng, C. L., Ma, C. P., and de Groat, W. C. (1993). Effects of capsaicin on micturition and associated reflexes in rats. Am. J. Physiol. 265, R132-R138.

Chizh, B. A., Headley, P. M., and Paton, J.F. (1997). An arterially-perfused trunk- hindquarters preparation of adult mouse in vitro. J. Neurosci. Methods 76, 177-182.

Clemens, J. Q. (2010). Basic bladder neurophysiology. Urol. Clin. North Am.37, 487-494.

Conte, B., D'Aranno, V., Santicioli, P., Giuliani, S., Mancinelli, A., Furio, M. Maggi, C.A., and Meli,A. (1988). New method for recording cystometrograms in conscious, freely moving rats. J. Pharmacol. Methods 19, 57-61.

Daly, D., Rong, W., Chess-Williams, R. Chapple, C., and Grundy, D. (2007). Bladder afferent sensitivity in wildtype and TRPV1 knockout mice. J. Physiol. 583, 663-674.

Das, A., Chancellor, M. B., Watanabe, T., Sedor, J., and Rivas, D. A. (1996). Intravesical capsaicin in neurologic impaired patients with detrusor hyperreflexia. J. Spinal. Cord Med. 19 , 190-193.

de Groat, W. C. (2006). Integrative control of the lower urinary tract: preclinical perspective. Br. J. Pharmacol. 147(Suppl. 2), S25-S40.

de Groat, W.C., and Yoshimura, N. (2010) Changes in afferent activity after spi- nal cord injury. Neurourol. Urodyn. 29, 63-76

de Seze, M., Wiart, L., de Seze, M. P., Soyeur, L., Dosque, J. P., Blajezewski, S., Moore, N., Brochet, B., Mazaux, J. M., Barat, M., and Joseph, P. A. (2004). Intravesical capsaicin versus resiniferatoxin for the treatment of detrusor hyperreflexia in spinal cord injured patients: a double-blind, randomized, controlled study. J. Urol. 171, 251-255.

Drake, M. J., Fowler, C. J., Griffiths, D. Mayer, E., Paton, J. F., and Birder, L. (2010). Neural control of the lower urinary and gastrointestinal tracts: supraspinal CNS mechanisms. Neurourol. Urodyn. 29, 119-127.

Ferguson, D. R., Kennedy, I., and Burton, T. J. (1997). ATP is released from rabbit urinary bladder epithelial cells by hydrostatic pressure changes - a possible sensory mechanism? J. Physiol. 505(Pt 2), 503-511.

Finney, S.M., Stewart, L.H., and Gillespie, J. I. (2008).Volume-induced responses in the isola,ted bladder: evidence for excitatory and inhibitory elements. BJU Int. 102, 1154-1161. 
Fowler, C. J. (2002). Bladder afferents and their role in the overactive bladder. Urology 59, 37-42.

Geirsson, G., Fall, M., and Sullivan, L. (1995). Clinical and urodynamic effects of intravesical capsaicin treatment in patients with chronic traumatic spinal detrusor hyperreflexia. $J$. Urol. 154, 1825-1829.

Gevaert, T., Vandepitte, J., Hutchings, G., Vriens, J., Nilius, B., and De Ridder, D. (2007). TRPV1 is involved in stretchevoked contractile changes in the rat autonomous bladder model: a study with piperine, a new TRPV1 agonist. Neurourol. Urodyn. 26, 440-450; discussion 451-443.

Gillespie, J. I., van Koeveringe, G. A., de Wachter, S. G., and de Vente, J. (2009). On the origins of the sensory output from the bladder: the concept of afferent noise. BJU Int. 103, 1324-1333.

Griffiths, D., Derbyshire, S., Stenger, A., and Resnick, N. (2005). Brain control of normal and overactive bladder. $J$. Urol. 174, 1862-1867.

Habler, H. J., Janig, W., and Koltzenburg, M. (1990). Activation of unmyelinated afferent fibres by mechanical stimuli and inflammation of the urinary bladder in the cat. J. Physiol. 425, 545-562.

Hawthorn, M. H., Chapple, C. R., Cock, M., and Chess-Williams, R. (2000). Urothelium-derived inhibitory factor(s) influences on detrusor muscle contractility in vitro. $\mathrm{Br}$. J. Pharmacol. 129, 416-419.

Kanai, A. J. (2011). Afferent mechanism in the urinary tract. Handb. Exp. Pharmacol. 202, 171-205.

Kanai, A., Roppolo, J., Ikeda, Y.,Zabbarova, I., Tai, C., Birder, L., Griffiths, D., de Groat, W., and Fry, C. (2007). Origin of spontaneous activity in neonatal and adult rat bladders and its enhancement by stretch and muscarinic agonists. Am. J. Physiol. Renal Physiol. 292, F1065-F1072.

Kasparov, S., and Paton, J. F. (1997). Changes in baroreceptor vagal reflex performance in the developing rat. Pflugers Arch. 434, 438-444.

Khadra, M. H., Satchell, P. M., and Vaughan, C. W. (1995). Sympathetic nervous system effects on feline bladder wall compliance throughout continence. Acta Physiol. Scand. 155, 31-39.

Kitta, T., Kakizaki, H., Furuno, T., Moriya, K., Tanaka, H., Shiga, T., Tamaki, N., Yabe, I., Sasaki, H., and Nonomura, K. (2006). Brain activation during detrusor overactivity in patients with Parkinson's disease: a positron emission tomography study. J. Urol. 175, 994-998.

Kreulen, D. L., Fredericks, C. M., and Anderson, G. F. (1977). Control of ureteral peristalsis during changes in intravesical pressure. Invest. Urol. 15 , 245-251.
Kruse, M.N., and De Groat, W.C. (1990). Micturition reflexes in decerebrate and spinalized neonatal rats. Am. J. Physiol. 258, R1508-R1511.

Kuru, M. (1965). Nervous control of micturition. Physiol. Rev. 45, 425-494.

Lehtoranta, M., Streng, T., Yatkin, E., Paranko, J., Kolts, I., Talo, A., and Santti, R. (2006). Division of the male rat rhabdosphincter into structurally and functionally differentiated parts. Anat. Rec. A Discov. Mol. Cell. Evol. Biol. 288, 536-542.

Levin, R. M., Ruggieri, M. R., Velagapudi, S., Gordon, D., Altman, B., and Wein, A. J. (1986). Relevance of spontaneous activity to urinary bladder function: an in vitro and in vivo study. J. Urol. $136,517-521$.

Maggi, C. A. (1991). Capsaicin and primary afferent neurons: from basic science to human therapy? J. Auton. Nerv. Syst. 33, 1-14.

Maggi, C. A., and Conte, B. (1990). Effect of urethane anesthesia on the micturition reflex in capsaicin-treated rats. $J$. Auton. Nerv. Syst. 30, 247-251.

Maggi, C. A., Giuliani, S., Santicioli, P., and Meli, A. (1986). Analysis of factors involved in determining urinary bladder voiding cycle in urethananesthetized rats. Am. J. Physiol. 251, R250-R257.

Mathias, C. J. (1991). Management of hypertension by reduction in sympathetic activity. Hypertension 17, III69-III74.

Mayer, E. A., Naliboff, B. D., and Craig, A. D. (2006). Neuroimaging of the braingut axis: from basic understanding to treatment of functional GI disorders. Gastroenterology 131, 1925-1942.

Ng, C. S., Herts, B. R., and Streem, S B. (2005). Percutaneous access to upper pole renal stones: role of prone 3-dimensional computerized tomography in inspiratory and expiratory phases. J. Urol. 173, 124-126.

Noto, H., Roppolo, J. R., Steers, W. D., and de Groat, W. C. (1991). Electrophysiological analysis of the ascending and descending components of the micturition reflex pathway in the rat. Brain Res. 549, 95-105.

Osman, F., Romics, I., Nyirady, P., Monos, E., and Nadasy, G. L. (2009). Ureteral motility. Acta Physiol. Hung. 96, 407-426.

Paton, J. F. (1996). A working heartbrainstem preparation of the mouse. J. Neurosci. Methods 65, 63-68.

Paton, J. F., Li, Y. W., Deuchars, J., and Kasparov, S. (2000). Properties of solitary tract neurons receiving inputs from the sub- diaphragmatic vagus nerve. Neuroscience 95, 141-153.

Paxinos, G., and Watson, C. (2007). The Rat Brain in Stereotaxic Coordinates. London: Elsevier Inc.
Pickering, A. E., and Paton, J. F. (2006). A decerebrate, artificially-perfused in situ preparation of rat: utility for the study of autonomic and nociceptive processing. J. Neurosci. Methods 155, 260-271.

Pickering,A.E.,Simms, A.E., and Paton,J.F. (2008). Dominant role of aortic baroreceptors in the cardiac baroreflex of the rat in situ. Auton. Neurosci. 142, 32-39.

Pickering, A. E., Waki, H., Headley, P. M., and Paton, J. F. (2002). Investigation of systemic bupivacaine toxicity using the in situ perfused working heartbrainstem preparation of the rat. Anesthesiology 97, 1550-1556.

Potts, J. T., and Paton, J. F. (2006). Optical imaging of medullary ventral respiratory network during eupnea and gasping in situ. Eur. J. Neurosci. 23, 3025-3033.

Sadananda, P., Chess-Williams, R., and Burcher, E. (2008). Contractile properties of the pig bladder mucosa in response to neurokinin A: a role for myofibroblasts? Br. J. Pharmacol. 153 , 1465-1473.

Santicioli, P., and Maggi, C. A. (1998). Myogenic and neurogenic factors in the control of pyeloureteral motility and ureteral peristalsis. Pharmacol. Rev. 50, 683-722.

Sasaki, M. (2005). Role of Barrington's nucleus in micturition. J. Comp. Neurol. 493, 21-26.

Simms, A. E., Paton, J. F., and Pickering, A. E. (2006). Disinhibition of the cardiac limb of the arterial baroreflex in rat: a role for metabotropic glutamate receptors in the nucleus tractus solitarii. J. Physiol. 575, 727-738.

Simms, A. E., Paton, J. F., and Pickering, A E. (2007). Hierarchical recruitment of the sympathetic and parasympathetic limbs of the baroreflex in normotensive and spontaneously hypertensive rats. J. Physiol. 579, 473-486.

Smith, J. C., Abdala, A. P., Koizumi, H., Rybak, I. A., and Paton, J. F. (2007). Spatial and functional architecture of the mammalian brain stem respiratory network: a hierarchy of three oscillatory mechanisms. J. Neurophysiol. 98, 3370-3387.

Streng, T., Santti, R., Andersson, K. E., and Talo, A. (2004). The role of the rhabdosphincter in female rat voiding. BJU Int. 94, 138-142.

Sugaya, K., and de Groat, W. C. (2002). Inhibitory control of the urinary bladder in the neonatal rat in vitro spinal cord-bladder preparation. Brain Res. Dev. Brain Res. 138, 87-95.

Szigeti, G. P., Somogyi, G. T., Csernoch, L., and Szell, E. A. (2005). Agedependence of the spontaneous activity of the rat urinary bladder. J. Muscle Res. Cell. Motil. 26, 23-29.

Tai, C., Wang, J., Jin, T., Wang, P., Kim, S. G., Roppolo, J. R., and de Groat, W. C. (2009). Brain switch for reflex micturi- tion control detected by FMRI in rats. J. Neurophysiol. 102, 2719-2730.

Taniguchi, N., Miyata, M., Yachiku, S., Kaneko, S., Yamaguchi, S., and Numata, A. (2002). A study of micturition inducing sites in the periaqueductal gray of the mesencephalon. J. Urol. 168, 1626-1631.

Thor, K. B., and de Groat, W. C. (2010). Neural control of the female urethral and anal rhabdosphincters and pelvic floor muscles. Am. J. Physiol. Regul. Integr. Comp. Physiol. 299, R416-R438.

Tian,L.,Prior,C.,Dempster,J.,and Marshall, I. G. (1997). Hexamethonium- and methyllycaconitine-induced changes in acetylcholine release from rat motor nerve terminals. Br. J. Pharmacol. 122, 1025-1034.

Walters, R. D., McMurray, G., and Brading, A. F. (2006). Comparison of the urethral properties of the female guinea pig and rat. Neurourol. Urodyn. $25,62-69$.

Yoshida, A., Kageyama, A., Fujino, T., Nozawa, Y., and Yamada, S. (2010). Loss of muscarinic and purinergic receptors in urinary bladder of rats with hydrochloric acid-induced cystitis. Urology 76, 1017.e7-1017.e12.

Zhang, H., Reitz, A., Kollias, S., Summers, P., Curt, A., and Schurch, B. (2005). An fMRI study of the role of suprapontine brain structures in the voluntary voiding control induced by pelvic floor contraction. Neuroimage 24, 174-180.

Zvara, P., Wright, A. J., Roach, K., Ursiny, M., Shapiro, B., Dagrosa, L. M., Nelson, M. T., and Heppner, T. J. (2010). A non-anesthetized mouse model for recording sensory urinary bladder activity. Front. Neurol. 1:127. doi: 10.3389/fneur.2010.00127

Conflict of Interest Statement: The authors declare that the research was conducted in the absence of any commercial or financial relationships that could be construed as a potential conflict of interest.

Received: 13 March 2011; accepted: 18 April 2011; published online: 13 May 2011.

Citation: Sadananda P, Drake MJ, Paton Julian FR and Pickering AE (2011) An exploration of the control of micturition using a novel in situ arterially perfused rat preparation. Front. Neurosci. 5:62. doi: 10.3389/fnins.2011.00062

This article was submitted to Frontiers in Autonomic Neuroscience, a specialty of Frontiers in Neuroscience.

Copyright $\odot 2011$ Sadananda, Drake, Paton and Pickering. This is an open-access article subject to a non-exclusive license between the authors and Frontiers Media SA, which permits use, distribution and reproduction in other forums, provided the original authors and source are credited and other Frontiers conditions are complied with. 